Background and aim Osteomyelitis is an inflammation of the bone that is usually due to bacterial infection. There are limited data on osteoarticular infections in the state of Qatar. The objectives of this study were to describe the demographic, clinical presentation and microbiological culture result of acute osteomyelitis in children

Methods Aretrospective and descriptive study was conducted at main tertiary hospital. Children hospitalised in our paediatric department with acute osteomyelitis from January 2000 to December 2013 were included.

Results The study comprised 79 patients. Mean age of presentation was (5.7) years and (62\%) were male. (91\%) had acute osteomyelitis whereas (9\%) were classified as chronic. most common bones affected were Femur (39.2\%), Tibia (15.2\%) followed by Foot (11.4\%) and iliac bone (10.1\%). Fever higher than $38^{\circ}$ on admission was found in $(65.8 \%)$, joint pain $(60.8 \%)$ and limping (45.6\%). Tenderness on examination was present in $(82.3 \%)$ followed by joint swelling $(59.5 \%)$ and restricted joint movement (55.7\%). Nearly (69\%) of Blood culture were negative. the most causative organisms were methicillin-susceptible Staphylococcus aureus (9.1\%), methicillin-resistant Staphylococcus aureus (9.1\%) and Strep Pyognes in (3.9\%).

Conclusion Our study confirmed that Microbiology screening tends to be negative but, if positive, Staphylococcus species is likely to be isolated. the metaphysis of long bones lower femur and upper tibiaprone to osteomyelitis. Mono-therapy for bone infection might be beneficial to start initially.

\section{PO-0216 COMMUNITY ACQUIRED PNEUMONIA IN CHILDREN REVIEW ARTICLE}

M Amirrad. Pediatrics, AlBaraha Hospital MOH, Dubai, United Arab Emirates

\subsection{6/archdischild-2014-307384.871}

Community acquired pneumonia (CAP) remains a major cause of childhood morbidity and mortality worldwide. There is still discrepancy in the definition, clinical diagnosis, identification of the causative pathogen, and treatment of CAP.

Having a high index of suspicion, using basic clinical skills in history and examination, and good knowledge of local prevalence of the microorganism and its resistance can guide the clinician to reach the most probable etiologic diagnosis of CAP to then treat it accordingly; avoiding under treatment (with risk of complications) and overtreatment (with risk of emergence of antibiotic resistance).

In this article, different aspects of paediatric CAP in developing and developed countries are reviewed; with some focus on comparing guidelines for CAP's management. The difference between guidelines is shown, with a need to have local (national) guidelines considering the prevalence of the etiologic organisms and its antibiotic susceptibility to avoid the emergence of resistance.

\section{P0-0217 WITHDRAWN}

\section{PO-0218 AUDIT OF EARLY MANAGEMENT OF CASES OF SEPTIC ARTHRITIS IN CHILDREN}

E Waits, S Shivangee, P Sharma, S Bandi. Paediatrics, Leicester Royal Infirmary, Leicester, UK

10.1136/archdischild-2014-307384.872
Introduction Septic arthritis can occur at any site of the body, but commonly occur in the lower limbs, especially knee and hip joints. It may arise from direct inoculation or spread from contiguous disease, but the most common method is haematogenous spread. We audited cases of septic arthritis in children as it can have serious consequences if mismanaged.

Objectives We looked at the management of children presenting with suspected septic arthritis.

Method All children who had a discharge code of septic arthritis between 1/01/07 and 29/04/13 were included. A standard proforma was used for data collection which recorded details of symptoms, signs, investigations and treatment

Results 39 patients were coded as septic arthritis. On closer look 11 patients were wrongly coded which left with 28 patients to audit.

$100 \%$ had full joint examinations and an orthopaedic review. $100 \%$ had appropriate blood tests including blood culture. 26 patients (93\%) had a joint aspiration out of which $12(46 \%)$ had an aspirate before giving antibiotics. Staph aureus- 5 (18\%) was the most coomon bacteria isolated from the joint aspirate followed by Group A beta-haemolytic strep 2 (7\%), Group B Beta haemolytic strep 2 (7\%), Coliform bacilli- 2 (7\%), Strep mitis and coagulase negative staph- 1 (4\%) and Strep pneumonia- 1 (4\%).

$100 \%$ had appropriate empirical antibiotics. The duration of antibiotics was variable but included a combination of intravenous and oral antibiotics.

Conclusion The audit highlighted the areas for improvement:

Urgent orthopaediac review in all cases

Joint aspirate ideally before giving antibiotics

A stand-alone guideline for children with septic arthritis.

\section{PO-0219 WITHDRAWN}

\section{PO-0220 CLOSTRIDIUM DIFFICILE IN A TERTIARY PAEDIATRIC HOSPITAL}

S Bota, L Varandas, G Cordeiro-Ferreira, C Gouveia. Pediatric Infecciology Unit, Hospital Dona Estefânia CHLC, Lisboa, Portugal

\subsection{6/archdischild-2014-307384.873}

Background An increase in paediatric Clostridium difficile (CD) infection incidence has been reported. Yet, its epidemiology and treatment schedules are not certain. We aim to describe the CD incidence, clinical presentation, treatment and outcomes in a children tertiary hospital.

Methods Data from Clostridium difficile identified cases by enzyme immunoassay (EIA), during 2010 and 2013, in Hospital Dona Estefânia (Portugal).

Results Eleven cases were identified, 73\% during 2013. Three children less than 12 months old were excluded (probable colonisation). A median age of 8,7 years was observed, with a highest incidence between children older than 10 years. Six of the cases were not hospitalised. In $62 \%$, the only symptom was diarrhoea. Among the eight cases, five (62\%) received multiple antibiotics before the CD detection, three (37\%) had a gastrointestinal disease and three (37\%) had recently undergone surgery. Six children $(75 \%)$ received treatment with metronidazol and no complications or deaths were reported. Recurrence was observed in two cases, with one child having three subsequent $\mathrm{CD}$ infection episodes. 